\title{
Interoperability Supported by Enterprise Modelling
}

\author{
Frank Walter Jaekel ${ }^{1}$, Nicolas Perry ${ }^{2}$, Cristina Campos ${ }^{3}$, Kai Mertins ${ }^{1}$, and \\ Ricardo Chalmeta ${ }^{3}$ \\ ${ }^{1}$ Fraunhofer IPK, Pascal Str. 8-9, 10587 Berlin - Germany \\ \{Frank-Walter.Jaekel, kai.mertins\}@ipk.fhg.de \\ 2 Ecole Centrale de Nantes, IRCCyN, 1 rue de la no, 44321 Nantes - France \\ Nicolas.Perry@irccyn.ec-nantes.fr \\ ${ }^{3}$ Grupo de investigación en Integración y Re-Ingeniería de Sistemas (IRIS), Dep. de \\ Llenguatges i Sistemes Informàtics, Universitat Jaume I, 12071 Castelló, SPAIN, \\ \{camposc, rchalmet\}@uji.es
}

\begin{abstract}
The application of enterprise modelling supports the common understanding of the enterprise business processes in the company and across companies. To assure a correct cooperation between two or more entities it is mandatory to build an appropriate model of them. This can lead to a stronger amplification of all the cross-interface activities between the entities. Enterprise models illustrate the organisational business aspects as a prerequisite for the successful technical integration of IT systems or their configurations. If an IT system is not accepted because its usefulness is not transparent to the staff members, then it quickly loses its value due to erroneous or incomplete input and insufficient maintenance. This at the end results in investment losses.

The paper exemplifies the strengths, values, limitations and gaps of the application of enterprise modelling to support interoperability between companies. It illustrates a proposal for a common enterprise-modelling framework. This framework is presented in terms of problems to face and knowledge based methodological approach to help solving them. A specific application demonstrates enterprise modelling and the synchronisation between the models as prerequisite for the successful design of Virtual Enterprises.
\end{abstract}

\section{Introduction}

The implementation of information systems and new organisational structures into and between companies requires discussions between different stakeholders of the enterprise (e.g. process design experts, managers, process owners, IT experts). Therefore the modelling of enterprise processes including related information systems and organisational units is an essential step in the process of changing and improving enterprise structures. The target is to achieve a common understanding of requirements of a new system. This is true for big companies as well as for small and medium size enterprises (SME). Furthermore the enterprise modelling bridges the gap in transforming process organisation of an enterprise 
and the processes implemented within the IT systems. The complexity of the modelling approach increases if used across enterprise networks.

The modelling of enterprise business processes is growingly becoming a well known technique especially within big companies. Now also SMEs are forced by their customers to increase the transparency of their processes. Moreover, the need of IT support such as ERP systems increases for SMEs. The establishment of information systems within a company is a difficult task. Various applications of IT systems are not efficient because of a lacking acceptance on the user side and of deficits between the real process organisation of the enterprise and the support of the IT systems.

Experiences from industrial projects illustrate that companies which buy an IT system without a clear strategy for enterprise business process improvement and little knowledge regarding the organisational effects often fail in applying the software. Therefore, big companies as well as SMEs require a modelling approach to create an enterprise (business) process blue print for a successful implementation of IT systems. The model is oriented to and across process owners or stakeholders of operational and management departments. Consequently the description of the process structure and its relations to different resources such as organisational units, IT infrastructure, information exchange, etc. has to be easily understandable.

Enterprise modelling concerns awareness of enterprise cultural particularities. The goal is to answer the question: 'How to make different enterprise models interoperable made from different modelling methodologies languages and metamodels, modelling background and environment?'.

The topic is covered by the European INTEROP [1] Network of Excellence concerned with inter-operability research for networked enterprises applications and software, its goals, rationale and early results and by the European integrated project ATHENA [2](Advanced Technologies for Interoperability of Heterogeneous Enterprise Networks and their Applications).

To address a Common Enterprise Modelling Framework definition, the first step is to establish a common base of understanding of different modelling constructs across different modelling languages that is a common modelling language such as the INTEROP UEML approach. The second step is to take into account the different ways of representing the real world within the model content, including aspects such as cultural and regional differences both in enterprise way of working but also in the way to build models, different objectives driven models and so on.

\section{Models Across Organizations}

\subsection{Problems of Enterprise Modelling between Companies}

In the actual situation regarding enterprise modelling several modelling methods and tools are used in enterprises (Figure 1). For example, $\mathrm{MO}^{2} \mathrm{GO}[3]$ supporting the integrated enterprise modelling is preferred because of a fast and easy 
understandable modelling method across different stakeholders. GRAI [4]Tools are prioritised especially for modelling the decisional processes of an enterprise. ARIS [5] is popular for enterprise modelling especially in the IT domain and IT departments. METIS [6] supports a very flexible meta modelling and therefore a good adaptation of the user wishes according the modelling constructs. The information covered by these tools is similar. Therefore, to save the investment for method training and model elaboration an exchange of the information modelled within the different tools should be provided. In the first step within INTEROP this is a topic of the Unified Enterprise Modelling Language (UEML) [7].

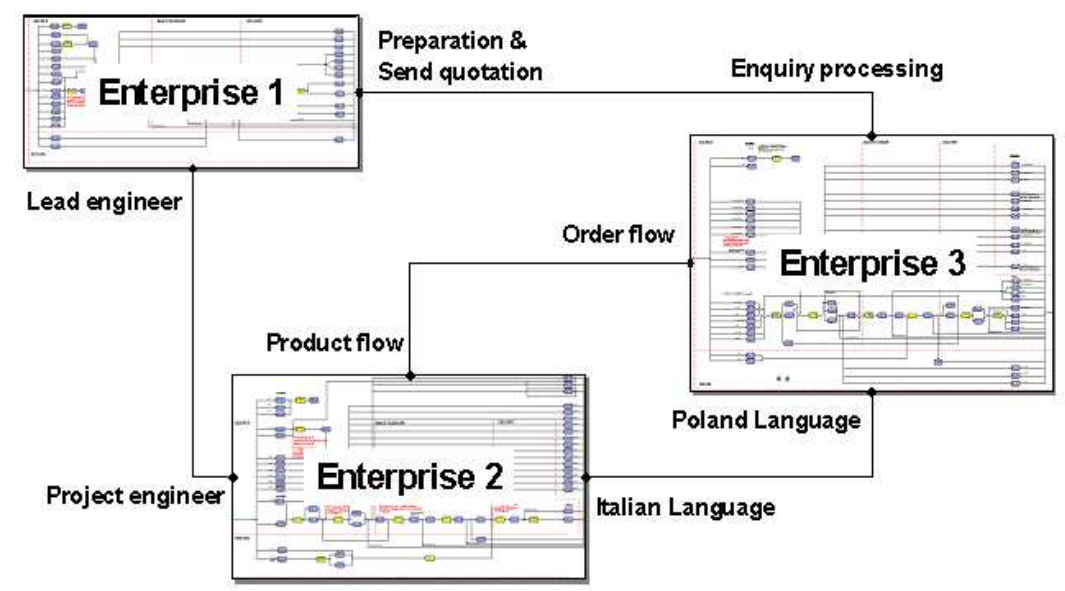

Fig. 1. Methods and Tools.

The problem of dealing with different models does not only depend on the modelling language. The same issue might be defined in two different models with different terms (e.g. Lead engineer / Project engineer) but at the same time in a third model these terms may have another meaning. Instead of the modelling language the natural language might be hindered by an information exchange and cooperated work on the models because a translation into an interlingua e.g. English might result in misinterpretations without having a common ontology support. The perspective between two models dealing with the same information might be different e.g. order processing or product process- 
ing concerning the external interfaces of an enterprise. The structuring of the processes as well as the design of the process chains might be dissimilar e.g. the two processes 'Preparation' and 'Send quotation' could appear in another model just the process 'Enquiry processing' (Figure 2). These are some examples of the problems under consideration in the INTEROP work around synchronisation of distributed enterprise models. Further problems arise concerning the management of such distributed enterprise models. An enterprise model associated with different other models requires clear procedures of how to perform changes [8].

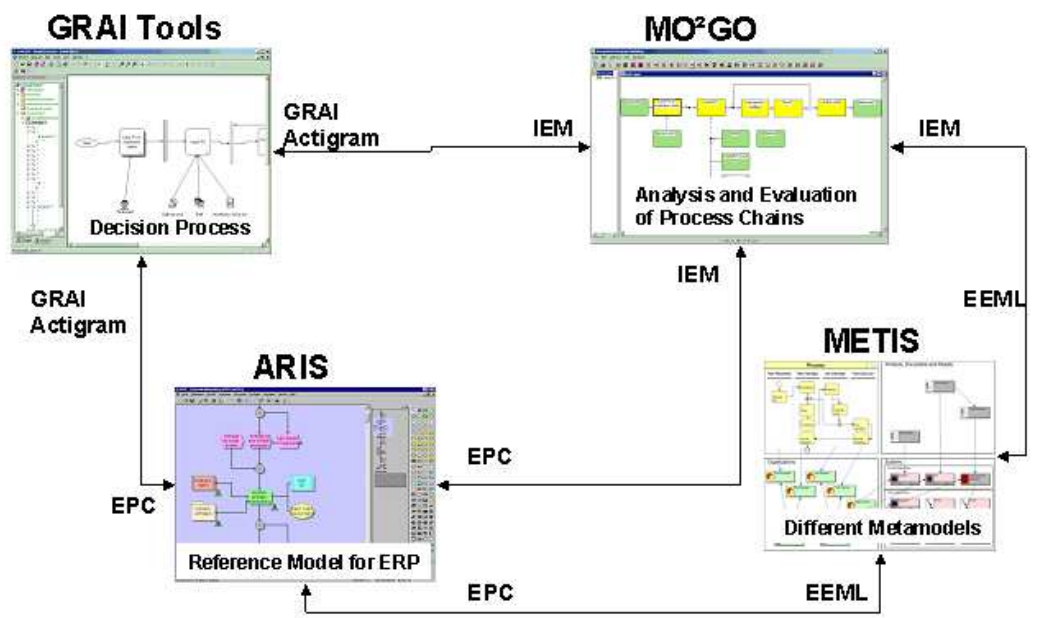

Fig. 2. Same modelling language but different understandings.

The intension of the paper is to motivate enterprise modelling supporting interoperability. More information regarding requirements and state of the art of enterprise modelling in the context of interoperability can be found in [9] [10] [11]

\subsection{Values of Enterprise Modelling}

The application of enterprise modelling supports the common understanding of the enterprise business processes in the company and across companies. The company is supported to succeed in reducing the throughput times, in improving the process quality, in reducing costs and therefore in improving the customer 
satisfaction and competitiveness. Enterprise modelling should be the basis of the information system planning process. The use of enterprise modelling for supporting and achieving company interoperability has different motivations, for example:

- Identification of companies' potentials for acting within different cooperation.

- Enabling companies to participate within collaborations through gathering the required data from the companies.

- Clarification of the connection between the operating processes of the companies and the required IT support through the illustration of additional operations.

- Model supported coordination, composition and synchronisation of organisation structures and business processes between the companies, especially identification of the aspects which support or, what is even more important, inhibit interoperability.

\section{Distributed Project Management and Models}

Lean extended enterprise and build-to-order induce better integration of Product Lifecycle Management activities that go through computer-aided systems and knowledge-based information environments. This change of landmarks from physical documents to electronics claims to redefine information support functionalities and knowledge-based tools to support worldwide project collaboration. Moreover, extended enterprises and collaborative projects have to analyse and control their core competencies to react efficiently to the market challenges (time to market, variability of products..).

The number of different enterprise concepts and complexities caused by different interpretations of these concepts encourages enterprises to standardise concepts and formalise behaviour. These efforts build re-usable and adaptable platforms and imply deep business architecture redeployments. The rapidly changing environment requires convenient collaboration and knowledge integration tools, and interoperability between different information sources. As a result, Knowledge Management has become a key facilitator in improving the global competitiveness of companies. As competitive drivers are forcing companies to innovate and change, effective knowledge management is essential to realise and also efficiently implement these changes.

To reach these goals, project management have both to integrate this expert's knowledge and the collaborative project constrains. Consequently interoperability, in terms of synchronisation problems, will occur at these two levels. We will mainly describe the first aspect assuming that a same approach can be applied for project models and alignment. 


\subsection{Experience feed back and method proposal}

Based on experimental cases we propose knowledge based projects methodology with three phases to optimise and ensure coherent enterprise project management:

- Project infrastructure definition, defines the fundamental elements of the project (domain infrastructure). Based on a syntax/semantic approach of the project's concepts model in order to differentiate concepts. The analysis of their relationship makes the domain architecture. Concepts 'behave' differently according to their context.

- Project architecture, explains the relationships between these elements and the way they are deployed (TO BE situation) in order to measure their efficiency.

- Document generation, describing the knowledge-based application specification for the software developer.

\section{$3.2 \quad$ Projects}

Next the two projects that integrate this methodology are described.

Information consolidation tool in order to build structured knowledgebased information environments This is a French National Project [12], including 5 academic partners, an aircraft manufacturer (the client) and a CADCAM System developer, in which we experimented different aspects of the distributed model management. Clear process definition, steps deliverables and objectives, concepts and working area had to be expressed. Based on the knowledge, experience and requeriments of the end user, the academic works had to capture and formalize the knowledge, improve and strengthen it before specifying software functionalities. We aimed to address 'efficiency of experts to share knowledge', and, due to our project team structure, we had to experiment it between partners. Indeed, specifications or constraints are usually transmitted from one expert to the other in a global convergence. The differences between their competencies limit the global understanding of problems. Computer integration in the expertise chain aims to optimise this kind of relations and thus the use of enterprise knowledge. The main difficulty encountered is to control the complexity of information quantity and informality. A harmonisation of the work-structure will reinforce the efficient use and clearness of information.

Economical model integration Software developers want to unify the four software solutions they developed independently [13]. The purpose is the cost estimation (costing) or sale price determination (pricing) in the micro-electronics field. The goal is to describe a generic economic model used for the determination of the product industrial value. Each tool answers various aspects from the silicon wafer to the finished products (electronic boards, mobile phone). The expert, 
distributed in different structures (production plant, design offices, buyer or seller services) had to unify parts of their costs models, their calculations rules, data inputs. The complexity relies on the fact that at different phases of the product life cycle, these software are used either by an engineer, a seller or a buyer. Consequently, their own integration of the tools in their project management is very different (objectives, information truth).

In both cases, we used a project modelling methodology supported by the MOKA supporting tool PC-Pack to build common ontology from expert documentation, the modelling tool MEGA, to perform Project Knowledge mapping with a systematic exploration, UML-like activity diagrams and UML class diagrams and time model synchronisation have been used as a possible support of these models alignment. The interest of these formalisms is that they propose a strong common syntax but let people free to rebuild their own semantic interpretation of meta-models thereby ensuring the whole project evolution in coherency with the initials objectives.

In term of model synchronisation, these two examples illustrated different distributed knowledge based projects, integrating cultural, geographical differences between partners or co-contractors. The spaces / domains comparison helps to analyse the model compliance (including model coherency, bijection, mapping of concepts, Meta model definition) and determine the synchronisation needs (this is a part of the domain infrastructure definition).

The use of a common methodology based on six core concepts (Syntax/Semantic: to give shareable modelled concepts, Infrastructure/Architecture: to define concepts and their interrelationships, Domain/Project: to represent 'AS IS' and 'TO BE' situation) helps the partners to understand each other and share models, information and requirements to perform the works. It still remains the problem of the life of such a distributed project model, for instance how to integrate new final user requirements on the knowledge based tool that rely on different fields of expertise after the end of the specification phase, or how to manage the evolution of the initial project objectives (financial support reduction and technical adaptations for example).

\section{Enterprise Modelling and Virtual Enterprises}

A Virtual Enterprise is a temporary alliance of independent enterprises that come together to share resources, skills and costs, with the support of the Information and Communication Technologies, in order to better attend market opportunities. To design an efficient and flexible Virtual Enterprise that gives the appearance of being a single enterprise to customers is a very complex task [14]. A Virtual Enterprise involves a great number of organizations, usually SME, that need to closely collaborate and to be in contact in order to achieve their objectives (competitiveness, better service for their clients, etc.) in this context the use of enterprise models is a key factor became successful. 


\subsection{Methodology for developing a virtual enterprise}

In order to help the creation and management of a Virtual Enterprise, partners develop models using different Enterprise Modelling Languages and different background knowledge. These enterprise models need to be interchangeable and understandable for people involved in each enterprise and for the whole virtual enterprise. In addition, Virtual Enterprises need to update their models due to the natural evolution of business, new legal requirements, changes in the strategy of the partners, and so forth. This kind of changes can affect concepts, business, results and other aspects in enterprise models that are needed to work correctly in real time [14]. Therefore synchronisation is really important in the process of setting up a Virtual Enterprise and it becomes more critical when a Virtual Enterprise is actually running.

The methodology for the virtual enterprise integration developed by IRIS group [15] shows how to set up practices and procedures in order to integrate a virtual enterprise. This methodology proposes (1) the definition of the conceptual aspects of the virtual enterprise and of each single enterprise (mission, vision, strategy, politics, and enterprise values); (2) the redesign of the new process map (internal business processes and cross-organisational business processes that are affected by changes), according to the previously defined concepts; (3) implementation of the VE new process map.; and (4) the extension of the information system (and the technological infrastructure) to support the process map of the virtual enterprise, considering the different levels of decision and the support technology. This methodology has been applied in several projects where thee adequate use of model languages and synchronisation between these models have been critical aspects to be successful.

These four points defined by the methodology are highly supported by the use of enterprise models, and consequently members in a virtual enterprise need to collaborate and to make al their enterprise models interchangeable and moreover, interoperable and synchronized. For the successful working on and integration of a Virtual Enterprises model contents and models management is a key issue to deal with. Therefore, it is necessary to establish the condition and the bases for good enterprise enterprise model management and synchronization.

\subsection{Needs for Enterprise Modelling in Virtual Enterprises}

The synchronization and management of enterprise models in a Virtual Enterprise is required under several aspects (e.g. model data, responsibilities, motivation, knowledge, configuration, social aspects etc.). The connection of different and distinct enterprise models is not limited to 'technical' or modelling language problems because, for instance, the same 'enterprise process' modelled with the same modelling language under the same objectives and requirements may differ whenever modelled by different persons.

As it is mentioned in this paper several modelling methods and tools are used in enterprises, and the application of these tools and models helps to common understanding. The same necessities analysed for enterprise interoperability can 
be found in the integration of a Virtual Enterprise and the same solutions must be taken into account. The application of the methodology developed by IRIS group demonstrates that the correct use of models is essential integration of a Virtual Enterprise.

Clear procedures stating how to manage and synchronize such models, templates and reference models, use of reference ontologies, training about models use and guidelines for modelling processes will help to increase the acceptance, use and results of enterprise models in the developing and management of a Virtual Enterprise and consequently improves the interoperability of all of its partners.

\section{Conclusions}

The reflections above illustrate the need of enterprise modelling to achieve and support interoperability between organisations. The INTEROP approach of the synchronisation and management of distributed enterprise models focuses on the organisational aspects of such models e.g. the common understanding regarding modelling structures or terms given to modelling elements to express the model content [7]. The INTEROP reference cases [11]illustrate the advantage, needs and requirements for enterprise modelling regarding interoperability as well. Most of the reference casess started with a modelling phase introducing different tools and methodologies. It also illustrates that there is not any general procedure applied and the models depend on how the organisation provides the modelling activities. Moreover, the involvement of the enterprise stakeholders is different. Under these circumstances one can imagine the problem of a company (e.g. a SME supplier) which has to participate into different co-operations (e.g.Virtual Enterprises) and has to be compliant with the other enterprise models. First of all, the modelling language might be considered. The solution could be using the INTEROP unified enterprise modelling language (UEML) approach [16] [17]. But afterwards the content of the model needs to be related to other models (structures, terms, etc.). Moreover, for achieving both compatibilities in the language and in the content (modelled information), the management of the decentralised models is required. What about changes within the model of the SME? Should they be reflected, directly, in all network models in which the SME participates? What are the results and implications of such changes?

Organizations develop models using different languages and different background knowledge. In order to achieve enterprise interoperability, it is necessary that these models will be interchangeable and comprehensible for people involved in the organization processes. The possibility of different companies to cooperate generates the necessity for models to be connected in a dynamic way. Changes in one of the models of an enterprise can affect processes, decisions and important aspects on the side of other partners. Therefore, synchronization is necessary among models from different enterprises in order to deal with changes, evolution and different views. This is a critical aspect (when models 
represent enterprise processes, information, organizational structures, products or decisions) for those who are closely connected to the same supply chain or to extended or virtual enterprises. methods have to be elaborated in order to support the model synchronisation and the decentralised usage of these models.

\section{Acknowledgments}

The presented research activities are partially supported by the European Network of Excellence IST-508011 'Interoperability Research for Networked Enterprises Applications and Software' INTEROP [1] and by CICYT DPI2003-02515.

\section{References}

1. INTEROP: Interoperability Research for Networked Enterprises Applications and Software NoE (IST-2003-508011). http://www.interop-noe.org (2005)

2. ATHENA: Advanced Technologies for Interoperability of Heterogeneous Enterprise Networks and their Applications) Project (IST-2003-2004). http://www.athenaip.org (2005)

3. $\mathrm{MO}^{2} \mathrm{GO}:$ Website. http://www.moogo.org (2005)

4. GRAITools: Website. http://www.graisoft.com (2005)

5. ARIS: Architecture of Integrated Information Systems. http://www.aris.org (2005)

6. Metis: Website. http://www.trouxmetis.com (2005)

7. Berio, G., Mertins, K., Jaekel, F.W.: Common Enterprise Modelling Framework for Distributed Organisations. In: 16th IFAC World Congress, Prague (Czech Republic) (2005)

8. SPIDER-WIN: Supply Information Dynamic Exchange and ContRol by Webbased Interaction Network. http://www.spider-win.de/spider-win.htm (2005)

9. Knothe, T., et al.: UEML Public Deliverable D2.3. http://www.ueml.org (2003)

10. Garcia, A.B.: ATHENA Public Deliverable D.A1.1.1 Enterprise Modelling Techniques and Technologies to Support Enterprise Interoperability. http://www.athena-ip.org (2004)

11. Jaekel, F.W., Piddington, C., Doumeingts., G.: INTEROP Public Deliverable D12.2 Deliverable 3.1. Reports on the Pilot Implementations and on the Possibility to Generalise the Methodology to Develop Take-up Actions towards SME's. http://www.interop-noe.org (2005)

12. Candlot, A., Perry, N., Bernard, A., Khodja, S.A.: Deployment of an Innovative Resource Choice Method for Process Planning. In: CIRP International Seminar on Manufacturing Systems, Florianapolis, (Brasil) (2005)

13. Perry, N., Mauchand, M., Bernard, A.: Integration of Cost Models in Design and Manufacturing. In: Advances in Design, Springer Series in Advanced Manufacturing, H. ElMaraghy and W. ElMaraghy (2005)

14. Chalmeta, R., Campos, C., Grangel, R.: References architectures for enterprises integration. The Journal of Systems and Software 57 (2001) 175-191 Elsevier.

15. Chalmeta, R., Grangel, R.: ARDIN Extension for Virtual Enterprise Integration. The Journal of Systems and Software (2003) Elsevier.

16. Berio, G., et al.: Deliverable 3.1. the UEML Project. http://www.ueml.org (2005)

17. Berio, G., Anaya, V., Ortiz, V.: Supporting Enterprise Integration Through a Unified Enterprise Modelling Language. In: EMOI Workshop, Riga, J. Grundspenkis and M. Kirikova (2004) 